\title{
Caseating lymphadenopathy at imaging: When ethnicity is deceptive
}

\author{
Dario Fabbri ${ }^{1}$, Chiara Baldovini ${ }^{2}$, Raffaele Pezzilli*1 \\ ${ }^{1}$ Department of Digestive System, Sant'Orsola-Malpighi Hospital, Bologna University, Bologna, Italy \\ ${ }^{2}$ Department of Experimental, Diagnostic and Specialty Medicine, Sant'Orsola-Malpighi Hospital, Bologna University, Bologna, \\ Italy
}

Received: March 28, 2016

DOI: $10.5430 /$ crim.v3n2p36
Accepted: May 3, $2016 \quad$ Online Published: May 4, 2016

URL: http://dx.doi.org/10.5430/crim.v3n2p36

\begin{abstract}
Background: Lymph node enlargement represents a clinical challenge in clinical practice and the underlying diagnosis represents the cornerstone of adequate treatment.

Case report: A caseating-like mediastinal lymph node investigated using computer tomography (CT) was found in an adult male patient coming from Pakistan, and a diagnosis of sarcoidosis was made only on a pathological specimen obtained by transbronchial needle aspiration.

Conclusion: To the best of our knowledge, caseating lymph-nodes have been never reported in sarcoidosis. It is important to note that only the presence of the typical aspect of sarcoidosis in the pathological specimen of an apparent caseating lymph node seen at imaging permits the real diagnosis of sarcoidosis especially in those patients in whom infectious disease is prevalent.
\end{abstract}

Key Words: Sarcoidosis, Tuberculosis, Computed Tomography, Pathology

\section{INTRODUCTION}

Lymph node enlargement represents a challenge in clinical practice and the underlying diagnosis represents the cornerstone of adequate treatment. Characterized by migration from developing countries to Western countries, tuberculosis has affected poor and vulnerable populations, especially in recent years; migrants are a key affected population, and migration as a social determinant of health increases tuberculosisrelated morbidity and mortality for migrants and their communities along all migration pathways. ${ }^{[1]}$ Sometimes, other diagnoses of lymph node enlargement may go unrecognized in this group of patients, and sarcoidosis is a good example of this misdiagnosis. Sarcoidosis is a chronic systemic disease and its etiology is unknown and prognosis varies greatly; usually its affects young adults, and the most common presentation is the presence of bilateral hilar lymphadenopathy associated with pulmonary infiltrates. ${ }^{[2]}$ Ocular involvement, represented mainly by uveitis and skin lesions, may also be present. ${ }^{[2]}$ The diagnosis is based on characteristic clinicalradiological features confirmed by the presence of epithelioid cell granulomas at histology and when other causes of granulomatous inflammation are excluded. ${ }^{[2]}$ The case of an adult male patient coming from Pakistan in whom the caseatinglike mediastinal lymph node investigated using computer tomography (CT) was found, and a diagnosis of sarcoidosis made is herein reported. To the best of our knowledge, caseating lymph nodes have never been reported in sarcoidosis, and our group believes that this case is worthy of being

\footnotetext{
*Correspondence: Raffaele Pezzilli; Email: raffaele.pezzilli@aosp.bo.it; Address: Department of Digestive System, S.Orsola-Malpighi Hospital, Via Massarenti 9, Bologna 40138, Italy.
} 
reported.

\section{CASE REPORT}

A 54-year-old Pakistan man was admitted to our Internal Medicine Unit in March 2015 for a persistent cough, sore throat, chest pain exacerbated by attacks of coughing and profuse sweating. He had migrated to Italy in 1989, working as a waiter, and he reported having an uncle, in Pakistan, with a previous diagnosis of tuberculosis. On admission, clinical examination of the abdomen, and the cardiac and pulmonary systems was unremarkable, and his vital signs were normal. Laboratory tests showed a slight leukocytosis (white cell count: $12,000 / \mathrm{mmc}$, neutrophils $77 \%$ ) and an increase in the inflammatory indices (C-reactive Protein: $7.09 \mathrm{mg} / \mathrm{dl}$, n.v. $<0.80$; erythrocyte sedimentation rate: $26 \mathrm{~mm}, \mathrm{n} . \mathrm{v} .<$ 20); blood cultures were negative. Beta- 2 microglobulin was slightly increased $(3.5 \mathrm{mg} / \mathrm{L}$, n.v. $<2.0)$, as was total $\operatorname{IgE}$ (125 UI/ml, reference value < 100) and serum CD4+/CD8+ ratio $(3.06 \%$, reference range $1.00-2.70)$; the antinuclear antibodies were border-line (1:80) having a speckled pattern. Rheumatoid factor, C3, C4, IgA, IgM, IgG and lactate dehydrogenase serum concentrations were normal, and serologic tests for toxoplasma infection, cytomegalovirus and Epstein-Barr virus were also negative. As shown in Figure 1 , a chest X-ray showed a diffuse thickening of the interstitial plot, associated with an increase in size of the hilar shadows and mediastinal profile due to lymphadenopathy. A thoracic and abdominal CT showed multiple enlarged lymph nodes at the mediastinum, the majority being confluent, some being colliquate (see Figure 2), a thickening of the septa, and centrilobular micro-nodules and multiple subcentimeter lymph nodes in the retroperitoneal area and in the mesentery. In addition, as shown in Figure 3, a nodule of the lingula of the lung was also detected. Thus, the differential diagnosis was between lymphoma, tuberculosis and sarcoidosis. The patient underwent ${ }^{18} \mathrm{~F}$-fluorodeoxyglucose (FDG) positron emission tomography which confirmed the presence of multiple and diffuse hypermetabolic lymphadenopathy in the mediastinum, in the hilum, in the carenal and paratracheal region, and in the celiac and crural area. All these lesions had a maximum standardized uptake value (SUVmax) of 29 (see Figure 4). Interferon-gamma release assays (IGRAs; QuantiFERON-TB Gold, QIAGEN GmbH, Hilden, Germany) and a tuberculin skin test (TST) were negative while the serum angiotensin converting enzyme (ACE) levels were lower (4 U/L, n.v. 66-114). For these reasons. a flexible bronchoscopy with bronchoalveolar lavage (BAL) for microbiologic testing and transbronchial needle aspiration (TBNA) of the mediastinal lymph nodes for cytology and mycobacterial research was carried out. The microbiologi- cal test did not show acid-fast bacilli (AFB), and both the polymerase chain reaction (PCR) and the culture were negative for Mycobacterium tuberculosis infection. Cytological examination showed the presence of lymphocytes and micro and macro noncaseating granulomas with focal necrosis. The patient then underwent a video-assisted mediastinoscopy with multiple lymph node biopsies in the precarenal and right paratracheal regions. The pathological analysis confirmed the absence of neoplastic cells, fungine cells and acid-fast bacilli, and showed the presence of chronic granulomatous lymph nodes with epithelioid cells and focal necrosis (see Figure 5). A diagnosis of sarcoidosis was made, and the patient started steroid therapy with prednisone (40 mg per day for three months which is tapered to every-other-day six weeks) and healed rapidly (see Figure 6).

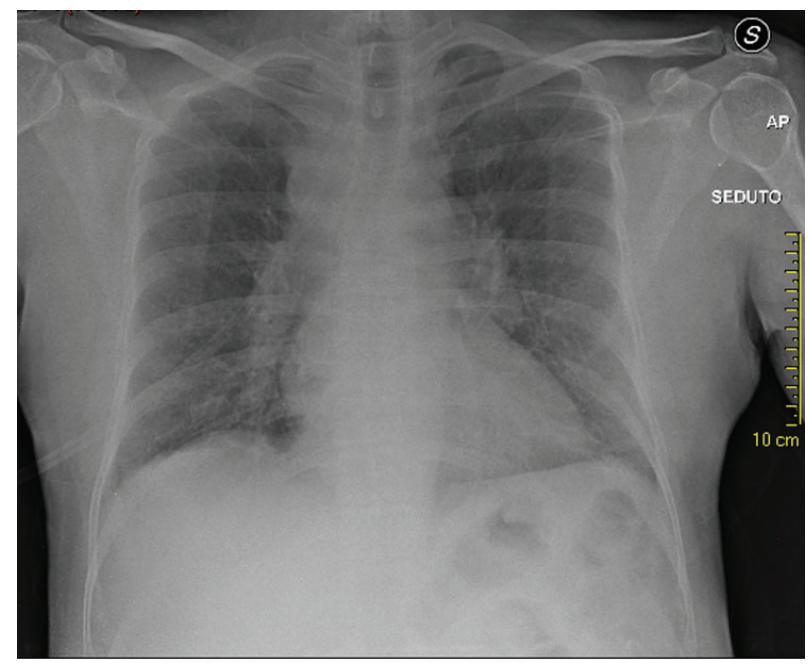

Figure 1. Chest X-ray showing a diffuse thickening of the interstitial plot, associated with an increase in size of hilar shadows and the mediastinal profile due to lymphadenopathy

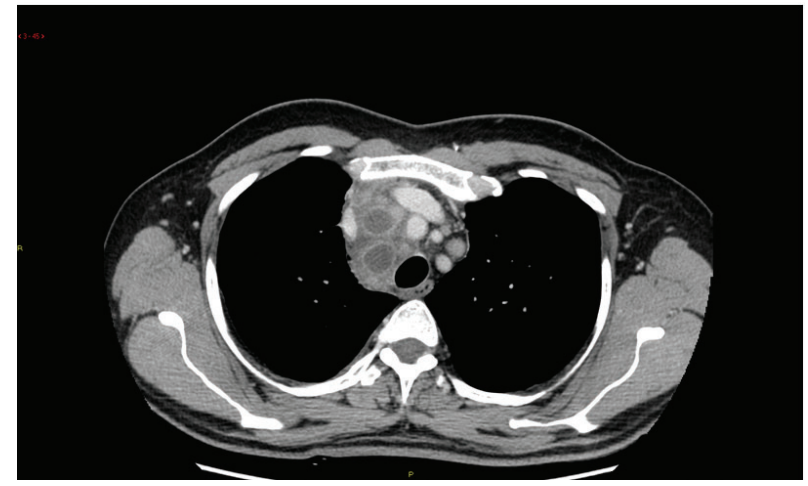

Figure 2. Thoracic computer tomography showing multiple enlarged lymph nodes at the mediastinum, the majority being confluent and some being colliquate 


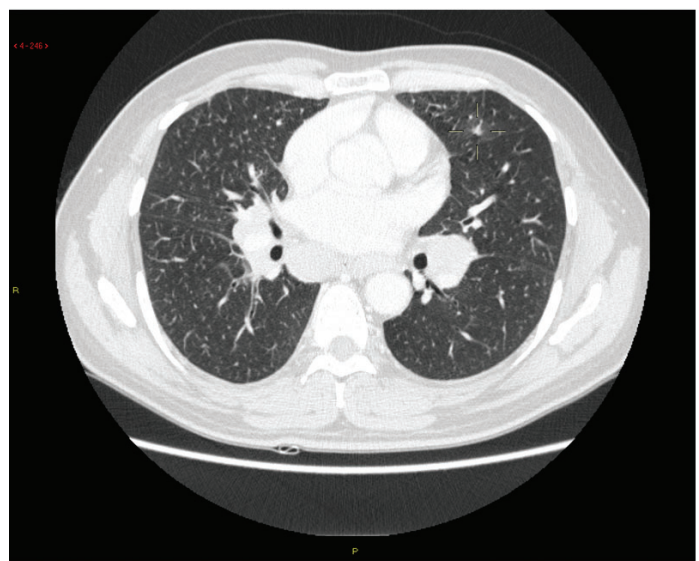

Figure 3. Thoracic computer tomography showing a nodule of the lingula

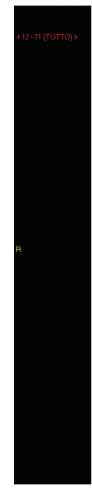

Figure 4. FDG-PET which confirmed the presence of multiple and diffuse hypermetabolic lymphadenopathy in the mediastinum, in the hilum, and in the carenal and paratracheal regions

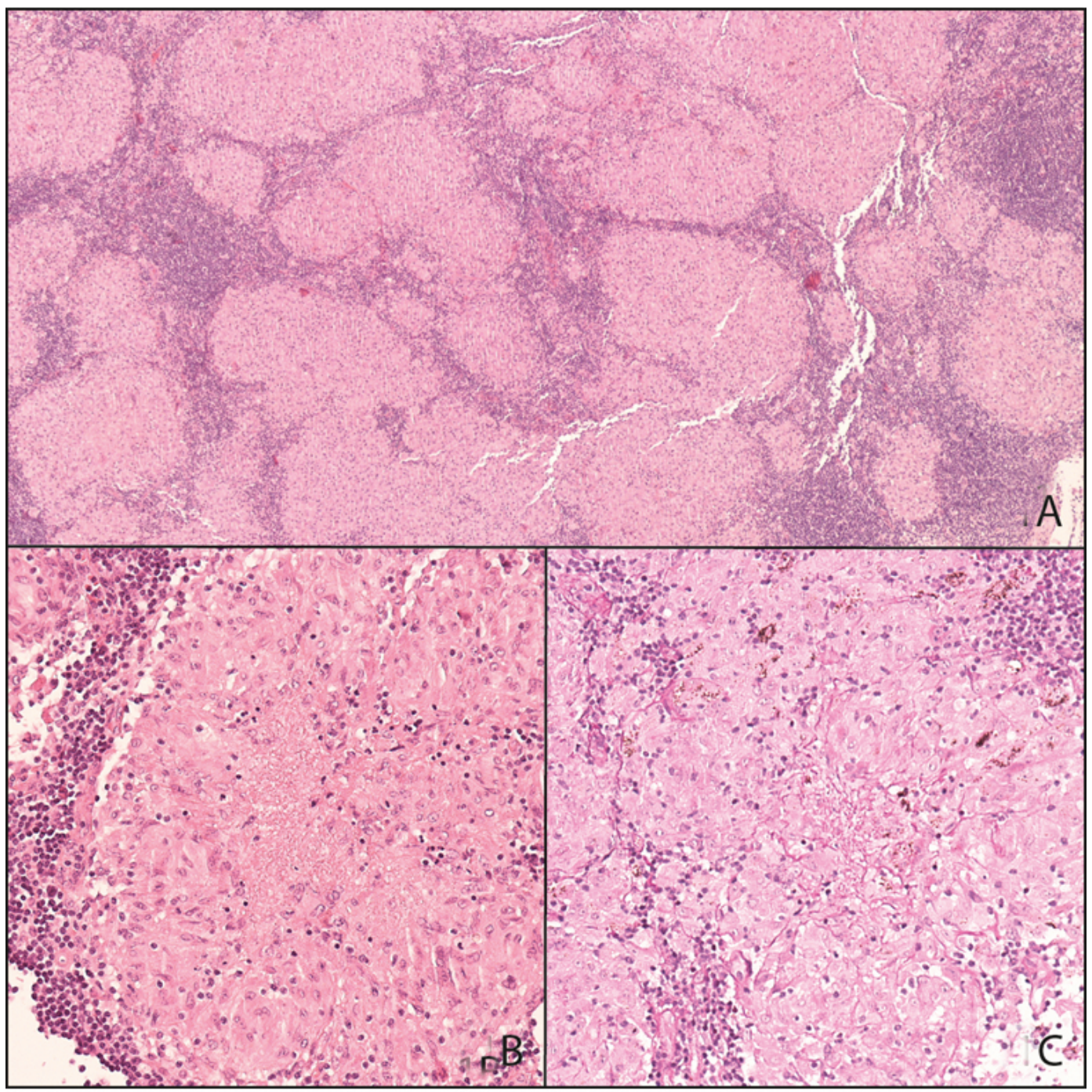

Figure 5. Panel a: $(4 \times ; H \& E)$ : numerous confluent granulomas, mainly composed of epithelioid cells in a lymphoid background. Panels b and c: $(20 \times ;$ H\&E, PAS-D): granulomas showing an area of necrosis consisting of a central fibrinoid focus 


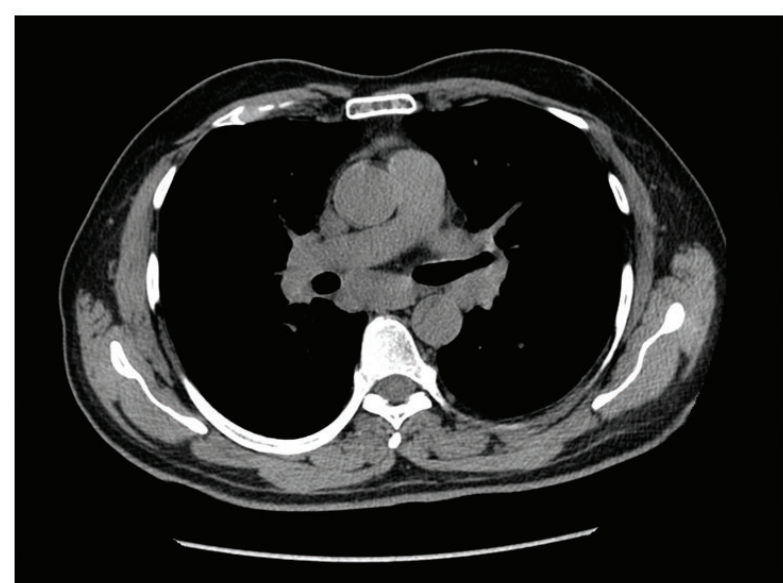

Figure 6. Thoracic computer tomography showing complete healing of the sarcoidodisis with the disapperarance of the multiple enlarged lymph nodes at the mediastinum

\section{Discussion}

Sarcoidosis is a systemic disease manifestation pathologically characterized by a chronic noncaseating granulomatous inflammation, usually involving the lung in more than $90 \%$ of the cases ${ }^{[3]}$ and affected patients may be asymptomatic. ${ }^{[4]}$

Sarcoidosis is progressive in $30 \%$ of cases, whereas may have a spontaneous regression in $40 \%-90 \%$ of patients. The mortality range from $1 \%$ to $5 \%$ of the cases mainly due to cardiorespiratory complications; an early diagnosis may be difficult when an elusive and non-specific onset is present. ${ }^{[5-9]}$

From a clinical point of view, the differential diagnosis was between tuberculosis, lymphoma or sarcoidosis. The fact that the patient came to Italy from a developing country and there was a familial history of tuberculosis, a suspicion of infective disease was compatible. In addition, the CT findings supported the diagnosis of infectious disease due to the presence of a suspected caseating lymph node. However, the absence of serology and isolation of Mycobacterium tuberculosis did not support the diagnosis of infectious disease. It is well known that $\mathrm{CT}$ is the standard technique for the diagnosis as well as the follow-up of sarcoidosis but it should be underlined that $\mathrm{CT}$ is often used for regional imaging of the chest and it also explain the low number of extrathoracic localizations detected. As in our case, abdominal lymphadenopathy is frequently detected in more than $70 \%$ of cases followed by lymph nodes located near the portal vein $(86 \%)$, the para-aortic region $(77 \%)$ and the coeliac axis $(59 \%) .{ }^{18} \mathrm{FDG}-\mathrm{PET} / \mathrm{CT}$ has recently been proposed for differentiating the solid lesions of the chest found at CT examination from malignant and benign lesions based on the ${ }^{18}$ FDG metabolism. ${ }^{[11]}{ }^{18}$ FDG accumulating within the sarcoid granuloma show the classical presence of a rich infiltrate of lymphocytes and macrophages ${ }^{[12,13]}$ even if ${ }^{18}$ FDG$\mathrm{PET} / \mathrm{CT}$ is not specific for sarcoidosis, and other pathological benign thoracic lesions showing ${ }^{18} \mathrm{FDG}$ uptake include infection, inflammation, granulomatosis and sarcoidosis. ${ }^{[14,15]}$

The appearance of lymph nodes at thoracic computer tomography is quite characteristic of sarcoidosis and in our case, multiple enlarged lymph nodes at the mediastinum were found, the majority being confluent which was compatible with sarcoidosis, but some lymph nodes were colliquate and this was not typcal of the disease. Thus, the findings of the ${ }^{18}$ FDG-PET/CT carried out were not conclusive, and only pathological examination of the lymph nodes involved permitted us to reach a diagnosis. Even if our patient had a suspicion of other diseases involving the lung, such as tuberculosis or lymphoma, only pathology of the specimens of the mediastinal lymph nodes obtained permitted us to reach a diagnosis of sarcoidosis. In conclusion, it is important to note that only the presence of the typical aspects of sarcoidosis in the pathological specimen of an apparent caseating lymph node seen at imaging permits the real diagnosis of sarcoidosis, especially in those patients in whom infectious disease is prevalent.

\section{CONFLicts OF INTEREST Disclosure}

The authors have declared no conflicts of interest.

\section{REFERENCES}

[1] Available from: http://www.who.int/tb/publications/WH OIOM_TBmigration.pdf

[2] Spagnolo P. Sarcoidosis: a critical review of history and milestones. Clin Rev Allergy Immunol. 2015; 49: 1-5. PMid:25737246 http://dx.doi.org/10.1007/s12016-015-8480-0

[3] Criado E, Sanchez M, Ramirez J, et al. Pulmonary sarcoidosis: typical and atypical manifestations at high-resolution CT with pathologic correlation. Radiographics. 2010; 30: 1567-1586. PMid:21071376 http://dx.doi.org/10.1148/rg. 306105512

[4] Iannuzzi MC, Rybicki BA, Teirstein AS. Sarcoidosis. N Eng J Med.
2007; 357: 2153-2165

[5] Cox CE, Davis-Allen A, Judson MA. Sarcoidosis. Med Clin N Am. 2005; 89: 817-828. PMid:15925652 http://dx.doi .org/10.10 $16 / j$.mcna. 2005.02.006

[6] Prabhakar HB, Rabinowitz CB, Gibbons FK, et al. Imaging features of sarcoidosis on MDCT, FDG PET, and PET/CT. AJR Am J Roentgenol. 2008; 190: S1-S6. PMid:18287458 http://dx.doi.o $\mathrm{rg} / 10.2214 / \mathrm{AJR} .07 .7001$

[7] Braun JJ, Kessler R, Constantinesco A, et al. ${ }^{18}$ FFDG PET/CT in sarcoidosis management: review and report of 20 cases. Eur $\mathbf{J}$ Nucl Med Mol Imaging. 2008; 35: 1537-1543. PMid:18418595 
http://dx.doi.org/10.1007/s00259-008-0770-9

[8] Hunninghake GW, Costabel U, Ando M, et al. Statement on sarcoidosis. The Joint Statement of the American Thoracic Society, the European Respiratory Society, and the World Association of Sarcoidosis and Other Granulomatous Disorders adopted by the ATS Board of Directors and by the ERS Executive Committee, February 1999. Am J Respir Crit Care Med. 1999; 160: 736-755.

[9] Loehrl TA, Smith TL. Inflammatory and granulomatous lesions of the larynx and pharynx. Am J Med. 2001; 111: 113S-117S. http://dx.doi .org/10.1016/S0002-9343(01)00856-7

[10] Silva M, Nunes H, Valeyre D, et al. Imaging of sarcoidosis. Clinic Rev Allerg Immunol. 2015; 49: 45-53. PMid:25726413 http: //dx.doi.org/10.1007/s12016-015-8478-7

[11] Lewis PJ, Salama A. Uptake of fluorine-18-Fluorodeoxyglucose in sarcoidosis. J Nucl Med. 1994; 35: 1647-1649. PMid:7931664
[12] Aide N, Benayoun M, Kerrou K, et al. Impact of ${ }^{18} \mathrm{~F}$ flurodeoxyglucose ([18F]-FDG) imaging in sarcoidosis: unsuspected neurosarcoidosis discovered by [18F]-FDG PET and early metabolic response to corticosteroid therapy. Br J Radiol. 2007; 80: e67-e71. PMid:17548505 http://dx.doi.org/10.1259/bjr/33076108

[13] Zhao S, Kuge Y, Kohanawa M, et al. Extensive FDG uptake and its modification with corticosteroid in a granuloma rat model: an experimental study for differentiating granuloma from tumors. Eur J Nucl Med Mol Imaging. 2007; 34: 2096-2105. PMid:17763849 http://dx.doi.org/10.1007/s00259-007-0529-8

[14] Alavi A, Buchpiguel CA, Loessner A. Is there a role for FDG PET imaging in the management of patients with sarcoidosis? J Nucl Med 1994; 35: 1650-1652. PMid:7931665

[15] Brudin LH, Valind SO, Rhodes CG, et al. Fluorine-18 deoxyglucose uptake in sarcoidosis measured with positron emission tomography. Eur J Nucl Med. 1994; 21: 297-305. PMid:8005153 http://dx.doi.org/10.1007/BF00947964 